\title{
Systematic celestial 4-configurations
}

\author{
Angela Berardinelli \\ Department of Mathematics, University of North Texas, Denton, Texas, USA
}

\section{Leah Wrenn Berman}

Department of Mathematics \& Statistics, University of Alaska Fairbanks, Fairbanks, Alaska, USA

Received 26 June 2012, accepted 20 June 2013, published online 15 November 2013

\begin{abstract}
Celestial 4-configurations are a class of highly symmetric geometric configurations of points and lines in the plane in which 4 points lie on each line and 4 lines pass through each point (that is, they are $\left(n_{4}\right)$ configurations). The set of isometries of the plane that map a configuration to itself (that is, the symmetries of the configuration) partition the points into orbits, called the symmetry classes of points, and likewise the symmetries of the configuration partition the lines into orbits as well, forming the set of symmetry classes of lines. Celestial 4-configurations have the property that two lines from each of two symmetry classes of lines pass through each point, and two points from each of two symmetry classes of points lie on each line; a celestial 4-configuration with $k$ symmetry classes is called $k$-celestial. Celestial configurations may be classified as being trivial, systematic, or sporadic. Previously, three non-trivial classes of 3-celestial 4-configurations were known. This paper presents a number of new systematic families of celestial 4-configurations, including 16 new 3-celestial families, four 4-celestial families, and three classes of $h$-celestial configurations for infinitely many values of $h$, although it does not provide a complete classification.
\end{abstract}

Keywords: Configurations, incidence geometry.

Math. Subj. Class.: 52C30, 51E30

E-mail addresses: AngelaBerardinelli@my.unt.edu (Angela Berardinelli), lwberman@alaska.edu (Leah Wrenn Berman) 


\section{Introduction}

A 4-configuration is a collection of points and straight lines, typically in the Euclidean or projective plane, so that each point has four lines passing through it and each line has four points lying on it; such configurations are often referred to as $\left(n_{4}\right)$ configurations, when the number $n$ of points and lines of the configuration is to be emphasized. If the configuration has non-trivial geometric symmetry, that is, if there exists a nontrivial isometry of the plane that maps the configuration to itself, then we say the configuration is symmetric, and the points and lines of the configuration may be partitioned into symmetry classes of points and of lines (i.e., the maximal orbit of a point under the symmetry group forms the symmetry class of that point, and similarly in the construction of the symmetry classes of lines). This usage of the word "symmetric" follows Grünbaum [14, p. 16] in reserving the word "symmetric" to refer to geometric properties of configurations. In other places in the literature (e.g. [7]), the word symmetric has been used to refer to $\left(n_{k}\right)$ configurations, which are 'symmetric' in the numbers of points and lines; again following Grünbaum, we shall call such configurations balanced, and reserve the use of 'symmetric' to emphasize geometric symmetry properties. If the number $n$ of points and lines is relevant, we refer to an $\left(n_{k}\right)$ configuration.

A 4-configuration is celestial if it has two points from each of two symmetry classes lying on each line and two lines from each of two symmetry classes of lines passing through each point; if there are $h$ total symmetry classes of points and lines, we refer to an $h$ celestial 4-configuration. Figure 1a shows an example of a 3-celestial 4-configuration with 21 points and lines: each point has two lines from each of two symmetry classes (indicated by color) passing through it, and each line has two points from each of two symmetry classes of points (again indicated by color) lying on it. Note that not all symmetric 4configurations are celestial, however; Figure 1 b shows a $\left(20_{4}\right)$ configuration with three symmetry classes of points and lines, with the property that one symmetry class of lines (shown in green) is incident with points from all three symmetry classes of points.

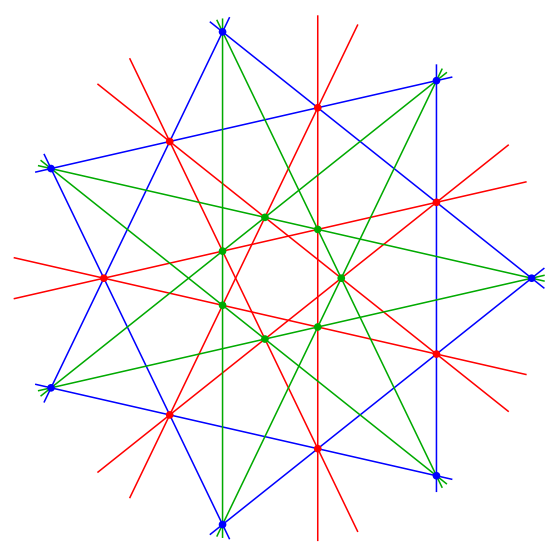

(a) $\mathrm{A}\left(21_{4}\right) 3$-celestial 4-configuration.

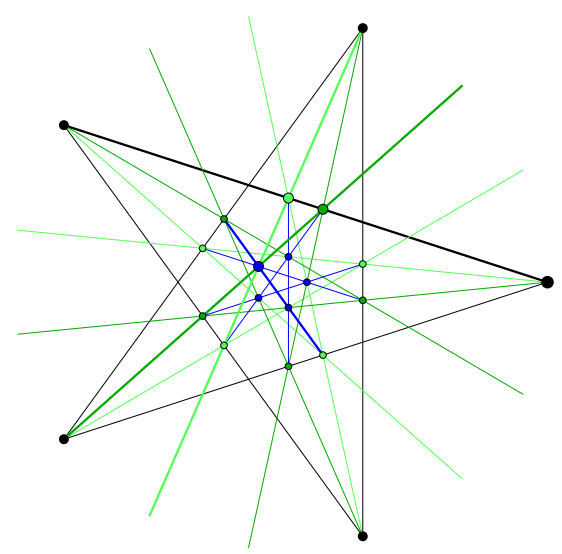

(b) A non-celestial $\left(20_{4}\right) 3$-astral 4-configuration, first shown in [13].

Figure 1: Examples of symmetric 4-configurations.

There has been a fair amount of investigation of 4-configurations in the past 20 years, 
beginning with Grünbaum and Rigby's 1990 discovery of an intelligible way of presenting a $\left(21_{4}\right)$ configuration [15]; this is the configuration shown in Figure 1a, which is the celestial 4-configuration with the fewest number of points and lines. In 2003, Marko Boben and Tomaž Pisanski introduced a class of highly symmetric configurations that they called polycyclic configurations which had rotational symmetry [8]. Celestial 4-configurations formed an important class of examples discussed in that paper.

Initial investigation into the classification of symmetric 4-configurations initially focused on astral configurations [1, 11] which have two symmetry classes of points and lines, two lines from each of two symmetry classes passing through each point, and two points from each of two symmetry classes lying on each line; that is, they are 2-celestial configurations. General celestial 4-configurations continued to be investigated as well [12], and a number of axioms were developed associating to each celestial configuration a configuration symbol (usually, several equivalent symbols). Given a potential configuration symbol, it is straightforward to determine if it corresponds to a configuration by verifying whether the axioms are satisfied. For this reason, celestial 4-configurations form the most well-understood class of 4-configurations and serve as building blocks for several other classes of configurations; for examples, see [2, 3, 4, 5, 6] (in some of those references, celestial configurations are referred to as $k$-astral configurations). However, despite their utility and the fact that there are concrete rules governing their existence, there has been very little work done in classifying $h$-celestial 4-configurations for $h>2$.

The most comprehensive description of celestial configurations occurs in Branko Grünbaum's recent monograph Configurations of Points and Lines [14]. In Section 1.5 of that reference, Grünbaum defines $h$-astral configurations to be configurations which have $h$ symmetry classes of points and $h$ symmetry classes of lines. We follow this usage. Unfortunately, in Sections 3.5 - 3.9 of the same reference, he refers to what we are calling celestial 4 -configurations as " $k$-astral" configurations, and all of his discussion of $k$-astral configurations in those sections refers to celestial configurations only. However, there are very nice classes of 4 -configurations that are $k$-astral (they have $k$ symmetry classes of points and lines) but not $k$-celestial; for example, see Figure $1 \mathrm{~b}$, in which one of the symmetry classes of lines is incident with points from three different symmetry classes of points (rather than points from only two symmetry classes of points). In this article, we use the term $k$-astral to refer to a configuration with $k$ symmetry classes of points and lines, whether celestial or not, and the term $k$-celestial to refer to a $k$-astral 4-configuration with the added property that every line contains two points from each of two symmetry classes and every point has two lines from each of two symmetry classes passing through it.

Following [14], celestial configurations are divided into three broad classes (discussed in more detail below): (1) trivial configurations; (2) systematic configurations, of which there are three known classes, and (3) sporadic configurations, which are non-trivial and (provably) non-systematic.

In this paper, we present a number of new families of systematic celestial 4-configurations. This answers affirmatively the following open question from Grünbaum [14, Section 3.7]: "Do there exist any other systematic families [of 4-celestial configurations] besides the ones listed above?" and also the open exercise 8, "Find some systematic families for [4-celestial] configurations other than the ones that arise from an $h$-celestial configuration with $h<k$ by insertion of matched pairs". 


\section{Celestial configurations}

The theory of celestial configurations has been developed over the past 20 years. The first known published pictures of celestial configurations appeared in [15] and as examples in a paper by Marušič and Pisanski [17], as well as in the paper on polycyclic configurations by Boben and Pisanski [8]; the configurations as a class were introduced by Grünbaum in unpublished course notes for a course on configurations [9]. The particular case of 2-celestial 4-configurations, also simply known as astral 4-configurations (in general, an $\left(n_{q}\right)$ configuration is astral if it has $\left\lfloor\frac{q+1}{2}\right\rfloor$ symmetry classes of points and lines, so a 4configuration is astral if it has 2 symmetry classes of points and lines), was considered in $[1,2,8,10,11]$; these configurations were completely classified in [1], with a more intelligible proof provided in [14, Section 3.6].

The most complete treatment of celestial 4-configurations, using the current terminology and approach, appears in Grünbaum's recent monograph on configurations, Configurations of points and lines [14, Section 3.5-3.8]. We follow his treatment in the following presentation (although, again, all his discussion in those sections refers to celestial configurations as $k$-astral configurations).

Every $h$-celestial $\left(n_{4}\right)$ configuration may be represented by a configuration symbol

$$
m \#\left(s_{1}, t_{1} ; s_{2}, t_{2} ; \ldots ; s_{h}, t_{h}\right),
$$

where $h$ is the number of symmetry classes of points, $n$ is the total number of points in the configuration, $m=\frac{n}{h}$ is the number of points in each symmetry class, and the $s_{i}$ and $t_{i}$ give the instructions for constructing the configuration geometrically.

Given points $P$ and $Q$ and lines $\ell_{1}$ and $\ell_{2}$, denote the line containing $P$ and $Q$ as $P \vee Q$ and the point of intersection of lines $\ell_{1}$ and $\ell_{2}$ as $\ell_{1} \wedge \ell_{2}$. If $w_{0}, w_{1}, \ldots, w_{m-1}$ form the vertices of a regular convex $m$-gon, labelled cyclically, then a line of span $s$ with respect to the $w_{i}$ is any line of the form $w_{i} \vee w_{i+s}$, and given the set of all lines $L_{i}=w_{i} \vee w_{i+s}$ of span $s$ with respect to $w_{i}$, the $t$-th intersection of the span $s$ lines is the set of points $L_{i} \wedge L_{i-t}$.

Given a configuration symbol $m \#\left(s_{1}, t_{1} ; s_{2}, t_{2} ; \ldots ; s_{h}, t_{h}\right)$, which is known to correspond to a configuration, that is, given a valid configuration symbol, the corresponding configuration may be constructed as follows.

1. Construct the vertices of a regular convex $m$-gon and label them cyclically as

$$
\left(v_{0}\right)_{0},\left(v_{0}\right)_{1}, \ldots,\left(v_{0}\right)_{m-1},
$$

and collectively as $v_{0}$. (Typically, $\left(v_{0}\right)_{i}=\left(\cos \left(\frac{2 \pi i}{m}\right), \sin \left(\frac{2 \pi i}{m}\right)\right)$ so that the points $v_{0}$ lie on the unit circle.)

2. Construct all lines of span $s_{1}$ with respect to the $v_{0}$ and label them collectively as $L_{0}$; in particular, $\left(L_{0}\right)_{i}=\left(v_{0}\right)_{i} \vee\left(v_{0}\right)_{i+s_{1}}$.

3. Construct a second set of points $v_{1}$ as the $t_{1}$-st intersection of the span $s_{1}$ lines $L_{0}$; that is, $\left(v_{1}\right)_{i}=\left(L_{0}\right)_{i} \wedge\left(L_{0}\right)_{i-t_{1}}$.

4. In general, lines $L_{j-1}$ are lines of span $s_{j}$ with respect to the points $v_{j-1}$, so that $\left(L_{j-1}\right)_{i}=\left(v_{j-1}\right)_{i} \vee\left(v_{j-1}\right)_{i+s_{j}}$, and points $v_{j}$ are the $t_{j}$-th intersection of the lines $L_{j-1}$, so that $\left(v_{j}\right)_{i}=\left(L_{j-1}\right)_{i} \wedge\left(L_{j-1}\right)_{i-t_{j}}$. 
For the configuration symbol corresponds to a valid configuration, the points with label $v_{h}$ (that is, the $t_{h}$-th intersections of the lines $L_{h}$ ) must correspond, as a set, to the points with label $v_{0}$, so that the construction closes up. That is, for the construction to be valid, the points $v_{h}$ constructed at the last step should have the same radius as the points $v_{0}$, they should have the same "angle"- the angle between point $\left(v_{h}\right)_{0}$ and $\left(v_{0}\right)_{0}$ should be an even multiple of $\frac{\pi}{m}$, and all sets of points and lines generated by the algorithm should be distinct. In addition, we want to avoid "extra incidences", where lines constructed in a certain step accidentally pass through points constructed several steps back.

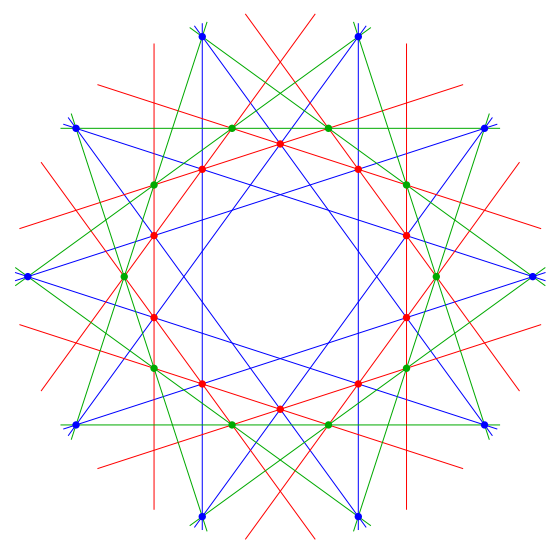

(a) symbol $10 \#(4,3 ; 1,2 ; 1,3)$

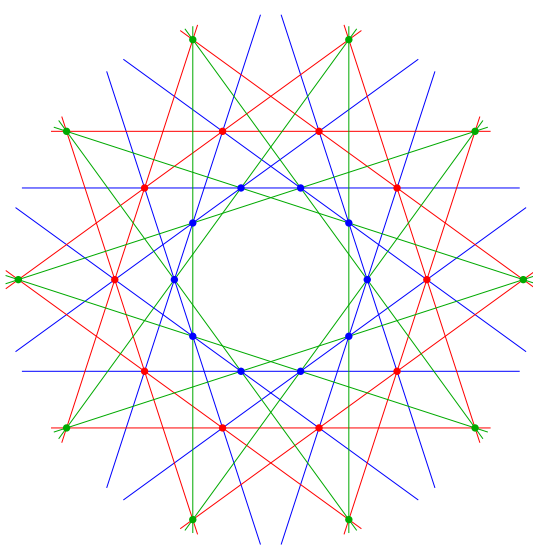

(b) symbol $10 \#\{1,3 ; 1,3 ; 4,2\}$

Figure 2: Two different 3-celestial 4-configurations. In each case, the points $v_{0}$ and lines $L_{0}$ are blue, the points $v_{1}$ and lines $L_{1}$ are red, and the points $v_{2}$ and lines $L_{2}$ are green.

Consequently, a configuration symbol $m \#\left(s_{1}, t_{1} ; \ldots ; s_{h}, t_{h}\right)$ with sequence $\left(s_{1}, t_{1} ; \ldots\right.$ $\left.; s_{h}, t_{h}\right)$ is valid if it satisfies the following four axioms:

(A1) (Even condition) The quantity $\frac{1}{2} \sum_{i=1}^{h}\left(s_{i}-t_{i}\right)$ is an integer.

This condition ensures that after following the configuration construction steps, the angle of the last set of points constructed coincides with the angles of the original set of points (rather than being offset by a factor of $\frac{\pi}{m}$ ).

(A2) (Order condition) No adjacent symbols in the sequence, taken cyclically, are equal: that is, $s_{i} \neq t_{i} \neq s_{i+1}$ for $i=1, \ldots, h-1$ and $s_{h} \neq t_{h} \neq s_{1}$.

This condition ensures that all symmetry classes of lines and points constructed are distinct.

(A3) (Cosine condition) $\prod_{i=1}^{h} \cos \left(\frac{s_{i} \pi}{m}\right)=\prod_{i=1}^{h} \cos \left(\frac{t_{i} \pi}{m}\right)$

This condition ensures that the radius of the last set of points constructed is equal to the radius of the original set of points.

Thus, satisfying (A1) and (A3) ensures that the points $v_{0}$ and the points $v_{h}$ coincide as sets. 
(A4) (Substring condition) No subsequence $s_{p}, t_{p} ; \ldots ; s_{q}$ or $t_{p} ; s_{p+1}, \ldots ; s_{q}, t_{q}$ may be completed to a sequence $s_{p}, t_{p} ; \ldots ; s_{q}, a$ or $a, t_{p} ; s_{p+1}, \ldots ; s_{q}, t_{q}$ which satisfies the previous rules (i.e., corresponds to a valid smaller configuration).

This is a technical condition to prohibit lines or points having extra, unwanted incidences.

Given a valid configuration symbol, any sequence formed with entries taken alternately without replacement from the set $S=\left\{s_{1}, s_{2}, \ldots, s_{h}\right\}$ and the set $T=\left\{t_{1}, \ldots, t_{h}\right\}$, or vice versa, will satisfy axioms (A1) and (A3); if they are ordered so as to satisfy axioms (A2) and (A4), then the new configuration symbol will also be valid. For example, the configuration shown in Figure 2a, with symbol $10 \#(4,3 ; 1,2 ; 1,3)$ has sets $S=\{4,1,1\}$ and $T=\{3,2,3\}$. If we construct the configuration symbol $10 \#\{1,3 ; 1,3 ; 4,2\}$, which satisfies axioms (A1) - (A4), the corresponding configuration also exists; see Figure $2 \mathrm{~b}$.

A valid configuration cohort refers to any symbol

$$
m \# S ; T
$$

where the multisets $S$ and $T$ satisfy axioms (A1) and (A3), for which there exists a sequence with entries alternately from $S$ and $T$ satisfying axioms (A2) and (A4). (Note that we explictly allow $S$ and $T$ to have repeated elements, but the order of the elements in the sets $S$ and $T$ is irrelevant.) If $|S|=|T|=h$, we may occasionally refer to an $h$-cohort; in this case, corresponding configurations are $h$-celestial. Given a cohort, every sequence with entries taken alternately from $S$ and $T$, or from $T$ and then from $S$, which satisfies (A2) and (A4) will correspond to a valid configuration symbol. Thus, in trying to classify celestial configurations, it is easier to classify configuration cohorts.

\subsection{Classification of cohorts}

If $S=T$, then the corresponding cohort is necessarily valid. Configuration cohorts of the form $m \# S ; S$ are called trivial. Some cohort sets fall into an infinite family. For example, the 2-celestial cohorts have been completely classified: there is one infinite family $6 q \#\{3 q-p, p\} ;\{2 q, 3 q-2 p\}$. A cohort which is a member of an infinite family is called systematic. Cohorts which are provably neither systematic nor trivial are called sporadic; for example, there are 15 sporadic 2-celestial cohorts [1],[14, Section 3.6]. Configurations whose corresponding cohorts are trivial/systematic/sporadic are also called triv$\mathrm{ial} /$ systematic/sporadic.

Definition 2.1. An $(h+j)$-cohort symbol

$$
m \#\left\{s_{1}, s_{2}, \ldots, s_{h}, a_{1}, a_{2}, \ldots, a_{j}\right\} ;\left\{t_{1}, t_{2}, \ldots, t_{h}, a_{1}, a_{2}, \ldots, a_{j}\right\}
$$

is reducible if and only if

$$
m \#\left\{s_{1}, s_{2}, \ldots, s_{h}\right\} ;\left\{t_{1}, t_{2}, \ldots, t_{h}\right\}
$$

is a valid $h$-cohort symbol.

A cohort which is not reducible is called primitive. 


\subsection{Determining valid cohorts}

In order to classify cohorts, it is useful to be able to generate, for a given $h$ and $m$, a list of all valid $h$-cohorts $m \# S ; T$.

Theorem 2.2. Given cohort symbol

$$
m \#\left\{s_{1}, \ldots, s_{h}\right\} ;\left\{t_{1}, \ldots, t_{h}\right\}
$$

with $h \geq 2$, the symbol violates (A2) (that is, every possible configuration sequence has at least two adjacent entries that are equal) if and only if there exists a $\in \cap T T$ that appears at least $h$ times in the list $\left(s_{1}, s_{2}, \ldots, s_{h}, t_{1}, t_{2}, \ldots, t_{h}\right)$.

Proof. $[\Longleftarrow]$ Let $a \in S \cap T$ be an element repeated $x$ times in the sequence

$$
\left(s_{1}, s_{2}, \ldots, s_{h}, t_{1}, t_{2}, \ldots, t_{h}\right),
$$

and assume $x \geq h$. Let $X$ count the number of times $a$ appears in $S$. To form a valid configuration symbol, it must be possible to construct a configuration sequence $\left(s_{1}, t_{1} ; s_{2}, t_{2} ; \ldots\right.$; $\left.s_{h}, t_{h}\right)$, relabelling the subscripts as necessary, so that no two adjacent elements are equal (with $s_{1}$ and $t_{h}$ considered to be adjacent). We may place all of the elements of $S$ into the sequence (in the appropriate slots) first. Then all positions adjacent to one of the $a$ s that have been placed from $S$ cannot be filled with one of the $(x-X)$ as from $T$. The placement that eliminates the fewest possible positions for the $a$ s in $\mathrm{T}$ is to place all the $a$ s from $S$ adjacent to each other in this way: $\left(a, t_{1} ; a, t_{2} ; \ldots ; a, t_{X} ; s_{X+1}, t_{X+1} ; \ldots ; s_{h}, t_{h}\right)$, so that there are $h-(X+1)$ slots in which as from $T$ could be placed (since there are $(X+1)$ slots $t_{i}$ which are blocked by the $a$ s). However, there are $(x-X) \geq(h-X)>(h-X-1)$ as which need to be placed, a contradiction.

[ $\Longrightarrow$ ] Assume every element in $S \cap T$ appears fewer than $h$ times in the sequence

$$
\left(s_{1}, s_{2}, \ldots, s_{h}, t_{1}, t_{2}, \ldots, t_{h}\right),
$$

and let $a$ be the element in $S \cap T$ which appears the most times in that sequence.

Case 1: $S \cap T=\emptyset$. Then

$$
\left\{s_{1}, t_{1} ; s_{2}, t_{2} ; \ldots ; s_{h}, t_{h}\right\}
$$

is a valid configuration sequence.

Case 2: $S \cap T \neq \emptyset$. Then there exists $a \in S \cap T$ which appears $x<h$ times in the list $\left(s_{1}, s_{2}, \ldots, s_{h}, t_{1}, t_{2}, \ldots, t_{h}\right)$. Suppose $a$ appears $X$ times in $S$. Then

$$
\left\{a, t_{1} ; a, t_{2} ; \ldots ; a, t_{X} ; s_{X+1}, a ; s_{X+2}, a ; \ldots ; s_{x}, a ; s_{x+1}, t_{x+1} ; \ldots ; s_{h}, t_{h}\right\}
$$

is a valid configuration sequence.

Corollary 2.3. If $S \cap T=\{a\}$, then $m \#\left\{s_{1}, s_{2}, \ldots, s_{h}, a\right\} ;\left\{t_{1}, t_{2}, \ldots, t_{h}, a\right\}$ is reducible to $m \#\left\{s_{1}, s_{2}, \ldots, s_{h}\right\} ;\left\{t_{1}, t_{2}, \ldots, t_{h}\right\}$.

Corollary 2.4. If $m \# S$; $T$ is $a(h+1)$-cohort and $\{a, b\} \subseteq S \cap T$ in which one of $a$ or $b$ appears at least $h$ times in the list $\left(s_{1}, s_{2}, \ldots, s_{h}, s_{h+1}, t_{1}, t_{2}, \ldots, t_{h}, t_{h+1}\right)$ then $m \# S ; T$ is not reducible.

Proof. Suppose $a$ appears at least once, and $b$ appears at least $h$ times. Attempt to reduce $m \# S ; T$ by removing one of the pairs of $a$ 's. The resulting cohort is not valid, by Theorem 2.2 . 


\subsection{2-celestial configurations}

2-celestial configurations have been completely classified (see [1] and [14, Section 3.6]). There is one infinite cohort:

$$
6 q \#\{3 q-p, p\} ;\{2 q, 3 q-2 p\}
$$

and 15 sporadic cohorts, shown in Table 1, plus disconnected multiples.

Table 1: Sporadic 2-celestial cohorts

\begin{tabular}{|c|c|}
\hline $30 \#\{7,1\} ;\{6,4\}$ & $30 \#\{11,1\} ;\{10,6\}$ \\
$30 \#\{12,2\} ;\{11,7\}$ & $30 \#\{13,7\} ;\{12,10\}$ \\
$30 \#\{14,6\} ;\{13,11\}$ & $30 \#\{8,2\} ;\{6,6\}$ \\
$30 \#\{12,6\} ;\{10,10\}$ & $30 \#\{13,1\} ;\{12,8\}$ \\
$30 \#\{14,4\} ;\{12,12\}$ & \\
\hline \hline $42 \#\{13,1\} ;\{12,6\}$ & $42 \#\{19,5\} ;\{18,12\}$ \\
$42 \#\{18,6\} ;\{17,11\}$ & \\
\hline \hline $60 \#\{22,2\},\{21,9\}$ & $60 \#\{27,3\},\{26,14\}$ \\
$60 \#\{25,5\},\{24,12\}$ & \\
\hline
\end{tabular}

In determining the list of all valid $h$-cohorts for a given $h$, the challenging axiom to verify is clearly (A3), since determining when products of sines or cosines are rational, or more generally, of solving trigonometric diophantine equations, is extremely challenging. The proof of the complete classification of 2-celestial configurations presented in [14], which is considerably less complex than that presented in [1], used results of Gerald Myerson [18] which determined all rational products of three and four sines of rational angles, and, more importantly for the current problem, determined all rational solutions to

$$
\sin \left(\pi x_{1}\right) \sin \left(\pi x_{2}\right)=\sin \left(\pi x_{3}\right) \sin \left(\pi x_{4}\right)
$$

which may be converted into the corresponding product of cosines using a simple trigonometric identity.

\section{New systematic families}

A fairly recent article by Miklós Laczkovich [16] apparently provides results on solutions to

$$
\sin \left(\pi p_{1}\right) \sin \left(\pi p_{2}\right) \sin \left(\pi p_{3}\right)=\sin \left(\pi q_{1}\right) \sin \left(\pi q_{2}\right) \sin \left(q_{3}\right),
$$

but its results are somewhat inexplicit and inaccessible for our current purposes.

For the remainder of this article, we will present known systematic families of $h$ celestial configurations - mostly for $h=3,4$ - which have been found primarily by ad hoc methods of analyzing lists of valid cohorts. For $h=3,4$ data were found by an exhaustive computer search using Mathematica. Essentially, for each $m$, we ran a simple incrementing loop on the discrete parameters

$$
1 \leq t_{3} \leq t_{2} \leq t_{1} \leq s_{3} \leq s_{2} \leq s_{1} \leq \frac{m}{2}
$$


which for each choice of discrete parameters checked whether it is trivial and whether it satisfies (A1), (A2) (using Theorem 2.2), and (A3); the expensive part of this process is checking the cosine condition. We collected all symbols that pass these tests. Then, using Corollaries 2.3 and 2.4 and the fact that all 2-celestial configurations are known, we eliminated reducible configurations. Finally, in the case of 3-cohorts, we removed alreadyclassified configurations. This left us with reasonably clean, sorted lists of all unclassified configurations for a given $m$. The generating Mathematica notebook and raw data are available at https://sites.google.com/a/alaska.edu/lwberman/, as well as lists of celestial configurations that are still unclassified. Production of the data was the main content of one of the author's [AB] Summer Fellows project at Ursinus College in 2009. We have generated data for 3-configurations for all $m \leq 120$ and for 4 -configurations for $m \leq 64$.

Traditionally, in the discussion of symbols for cohorts and configurations, the parameters $s_{i}$ and $t_{i}$ are chosen to be less than $m / 2$. However, in the description of infinite families of cohorts, it is often helpful to allow the parameters to take on larger values; we consider the geometry of the configuration construction to determine the appropriate way to reduce the symbol.

Suppose a regular $m$-gon has all diagonals of span $s$. Then a diagonal of span $s$ and of span $-s=m-s \bmod m$ correspond to the same line. Reflecting over a diameter gives a different diagonal, but of the same span. We define the standard form of a symbol element $s$ to be a number $s^{\prime}$ with $1 \leq s^{\prime}<\frac{m}{2}$ computed by doing the following:

1. First, take the absolute value of $s$

2. Then, reduce $s$ modulo $m$.

3. If $s \geq \frac{m}{2}$, replace $s$ with $m-s$; otherwise, $s$ is already between 1 and $\frac{m}{2}$.

In the presentations of the infinite families, the spans in question are not, typically, given in standard form, although the list of valid cohorts is generated in standard form (that is, we restrict the values of $s_{i}$ and $t_{i}$ to be positive and less than $\frac{m}{2}$ ).

\section{3-celestial families}

A number of years ago, one of the authors [LWB] found the following systematic cohorts of 3-celestial configurations (which were unpublished until their discussion in [14, Section 3.7]):

- $2 q \#\{q-p, p, q-2 r\} ;\{q-r, r, q-2 p\}$

- $3 q \#\{q+p, q-p, p\} ;\{q, q, 3 p\}$

- $10 q \#\{5 q-p, 2 p, p\} ;\{5 q-4 p, 4 q, 2 q\}$

At the time, these families appeared to be exhaustive for 3-celestial configurations $m \# S, T$ where $m$ was not divisible by 6 , and there was a conjecture that there were no other cohorts for such $m$. Very little was known about classification of 3-celestial configurations when $m$ was divisible by 6: one systematic family for $m=6 q$ was listed in [14, Section 3.7], but this family, $m \#\{3 q-p, r, p\} ;\{3 q-2 p, 2 q, r\}$, is reducible to the known family of 2 -celestial configurations. 
Subsequent computer experiments have revealed the existence of six 3-celestial cohorts for $m=70$. These have not yet been explained. In addition, there is a single unclassified cohort for $m=105$. These are listed in Table 2. Both 70 and 105 are divisible by 35, which raises the unexpected possibility that there is an infinite family for $m$ divisible by 35, but no such family is known. (It is not unreasonable that an infinite family of the form $m=$ $35 q$ would not have any entries for $q=1$; for example, the infinite family of 2-celestial configurations does not have any entries for $q=1$, since there are no configurations with $m=6$.

Table 2: Unclassified cohorts for $m=70$ and $m=105$.

$$
\begin{gathered}
m=70 \\
\{24,14,4\} ;\{20,19,9\} \\
\{28,12,2\} ;\{27,13,10\} \\
\{29,10,1\} ;\{26,16,14\} \\
\{30,17,3\} ;\{28,22,8\} \\
\{33,23,20\} ;\{32,28,18\} \\
\{34,14,6\} ;\{31,30,11\} \\
m=105 \\
\{45,30,15\} ;\{42,35,21\}
\end{gathered}
$$

However, a number of new systematic families of 3-celestial cohorts have been found in the case when $m$ is divisible by 6 , discussed in Section 5.1, below.

\section{3-celestial cohorts when $m$ is divisible by 6}

\section{$5.1 m=6 q$}

For $m$ divisible by 6 , the following families are known.

Family 1: $6 q \#\{2 q, q-p, 3 p\} ;\{2 q-p, q+2 p, p\}, p=1, \ldots, 2 q-1$

Family 2: $6 q \#\{3 q-p, 2 p, p\} ;\{2 q, 2 q, 3 q-4 p\}, p=1, . ., q-1$

Family 3: $6 q \#\{2 q+p, q-2 p, p\} ;\{2 q, q+p, 3 p\}, p=1, \ldots, 2 q-1$

Family 4: $6 q \#\{3 q-3 p, 2 p, p\} ;\{3 q-4 p, q+p, q-p\}, p=1,2, \ldots, 3 q-1$

Family 5: $6 q \#\{q+2 p, q-2 p, p\} ;\{q+p, q-p, 3 p\}, p=1, \ldots, 3 q-1$

Family 6: $6 q \#\{3 q-p, 6 p, p\} ;\{3 q-4 p, 2 q+2 p, 2 q-2 p\}, p=1, \ldots,\left\lfloor\frac{3 q}{2}\right\rfloor$

Family 7: $6 q \#\{2 p, 3 p, 3 q-3 p\} ;\{3 q-4 p, q-2 p, q+2 p\}, p=1, \ldots,\left\lfloor\frac{3 q}{2}\right\rfloor-1$

Family 8: $6 q \#\{3 q-2 p, q-2 p, q+2 p\} ;\{2 q, 3 q-3 p, 3 p\}, p=1, \ldots,\left\lfloor\frac{3 q}{2}\right\rfloor$

Family 9: $6 q \#\{3 q-3 p, 6 p, 3 p\} ;\{q+4 p, 3 q-4 p, q-4 p\}, p=1, \ldots,\left\lfloor\frac{q}{2}\right\rfloor$

Verification of the validity of these families proceeds by verifying the cosine condition, using standard trigonometric identities: the identity $\cos (a) \cos (b)=\frac{1}{2}(\cos (a+b)+\cos (a-$ 
$b))$ is especially useful. For example, to see that Family 1 works, note that in the cosine condition, if $\phi=\frac{\pi}{6 q}$,

$$
\begin{aligned}
\text { LHS } & =\cos (2 q \phi) \cos ((q-p) \phi) \cos (3 p \phi) \\
& =\cos \left(\frac{\pi}{3}\right) \cos ((q-p) \phi) \cos (3 p \phi) \\
& =\frac{1}{2} \cos ((q-p) \phi) \cos (3 p \phi) \\
& =\frac{1}{4}(\cos ((q-p+3 p) \phi)+\cos (((q-p)-3 p) \phi)) \\
& =\frac{1}{4} \cos ((q+2 p) \phi)+\frac{1}{4} \cos ((q-4 p) \phi) .
\end{aligned}
$$

On the other hand,

$$
\begin{aligned}
\text { RHS } & =\cos ((2 q-p) \phi) \cos ((q+2 p) \phi) \cos (p \phi) \\
& =[\cos ((2 q-p) \phi) \cos (p \phi)] \cos ((q+2 p) \phi) \\
& =\frac{1}{2}(\cos ((2 q-p+p) \phi)+\cos ((2 q-p-p) \phi)) \cos ((q+2 p) \phi) \\
& =\frac{1}{2}\left(\cos \left(\frac{2 q \pi}{6 q}\right)+\cos ((2 q-2 p) \phi)\right) \cos ((q+2 p) \phi) \\
& =\frac{1}{2}\left(\frac{1}{2}+\cos ((2 q-2 p) \phi)\right) \cos ((q+2 p) \phi) \\
& =\frac{1}{4} \cos ((q+2 p) \phi)+\frac{1}{2} \cos ((2 q-2 p) \phi) \cos ((q+2 p) \phi) \\
& =\frac{1}{4} \cos ((q+2 p) \phi)+\frac{1}{4}\left(\cos \left(\frac{3 q \pi}{6 q}\right)+\cos ((q-4 p) \phi)\right) \\
& =\frac{1}{4} \cos ((q+2 p) \phi)+0+\frac{1}{4} \cos ((q-4 p) \phi),
\end{aligned}
$$

so the cosine condition is satisfied. In practice, it is easier to let a computer algebra system verify the cosine condition.

Consider all $m \leq 120$ which are divisible by 6 but not 12 , that is,

$$
m=18,30,42,54,66,78,90,102,114 .
$$

For $m=18,54,78,102,114$ these nine families completely exhaust the experimentally derived configurations. For $m=30,42,90$ we expected that there would be additional configurations, because these are values of $m$ which yielded sporadic 2-celestial configurations, and this expectation was fulfilled.

Surprisingly, there is a collection of cohorts for $m=66$, given in Table 3 , in addition to the cohorts have already been identified as $2 q, 3 q$ and $6 q$ cohorts, which do not seem to be part of a systematic infinite family. However, they do satisfy the following family description:

$$
66 \#\{24 p, 12 p, 6 p\} ;\{11+6 p, 11+3 p, 2 \cdot 11-3 p\}, \quad p=1, \ldots, 10
$$

and

$$
66 \#\{24 p, 12 p, 6 p\} ;\{11+6 p, 2 \cdot 11,11-6 p\}, \quad p=1, \ldots, 5
$$


Table 3: The unexpected 3-celestial cohorts for $m=66$.

\begin{tabular}{l|l}
\multicolumn{2}{c}{$m=66$} \\
$\{24,12,6\} ;\{19,17,14\}$ & $\{30,12,6\} ;\{29,19,4\}$ \\
$\{24,12,6\} ;\{22,17,5\}$ & $\{30,18,6\} ;\{29,20,13\}$ \\
$\{24,18,12\} ;\{23,17,16\}$ & $\{30,18,6\} ;\{29,22,7\}$ \\
$\{24,18,12\} ;\{23,22,1\}$ & $\{31,7,2\} ;\{30,18,6\}$ \\
$\{25,8,5\} ;\{24,12,6\}$ & $\{31,22,13\} ;\{30,24,18\}$ \\
$\{28,5,1\} ;\{24,18,12\}$ & $\{31,23,10\} ;\{30,24,18\}$ \\
$\{30,12,6\} ;\{25,22,19\}$ & $\{32,13,1\} ;\{30,24,18\}$ \\
$\{30,12,6\} ;\{26,25,7\}$ &
\end{tabular}

These cohorts, along with the $2 q, 3 q$ and $6 q$ cohorts, exhaust the data for $m=66$ but they do not appear to obviously generalize to an additional $6 q$ family.

\section{$5.2 m=12 q$}

For 3-celestial configurations with $m$ divisible by 12 , in addition to the $6 q$ families discussed in the previous section, there are three known $12 q$ families:

- $12 q \#\{5 q, 3 p, q\} ;\{4 q+p, 4 q-p, p\}, p=1, \ldots, 2 q-1$

- $12 q \#\{5 q, q, 6 q-4 p\} ;\{6 q-p, 2 p, p\}, p=1, \ldots, 3 q-1$

- $12 q \#\{5 q, 6 q-4 p, q\} ;\{6 q-2 p, 3 q+p, 3 q-p\}, p=1, \ldots, q-1$

The $2 q, 3 q, 6 q$ and $12 q$ families completely exhaust the known data for all $m \leq 120$ except for the following cases.

- $m=66,70,105$ : conjectured sporadic cohorts (the 66 family is "classified" but does not appear to generalize)

- $m=30,60,90,120$ : there are four known $30 q$ families (see below), but there are more systematic families yet to be found.

- $m=42,84$ : almost certainly there are systematic families, but none have been found yet.

\section{$5.3 m=30,42,60$}

These are very complicated. Note that for 2-celestial configurations, sporadic configurations exist precisely when $m=30,42,60$. Thus additional families, and probably additional sporadic cases, are to be expected for these values.

Known familes for $m$ divisible by 30 are the following.

- $30 q \#\{15 q-p, 10 q, p\} ;\{15 q-2 p, 12 q, 6 q\}$

- $30 q \#\{15 q-p, 12 q, p\} ;\{15 q-2 p, 13 q, 7 q\}$

- $30 q \#\{15 q-p, 6 q, p\} ;\{15 q-2 p, 11 q, q\}$

- $30 q \#\{12 q, 6 q, 3 p\} ;\{10 q+p, 10 q-p, p\}$

These are not exhaustive. 


\section{4-celestial families}

The situation with 4-celestial configurations is considerably less well-understood than the 3 -celestial configurations. A few infinite families have been discovered, for $m$ divisible by $2,3,6,10$, but the data suggest that the complete story is not yet known.

In particular, unlike the case of 2-celestial and 3-celestial configurations, there are a few known 4-celestial configurations for prime $m$. A single cohort exists for $m=17$, and there are two cohorts for $m=61$. These are listed in Table 4.

Table 4: Known 4-celestial cohorts for prime $m$.

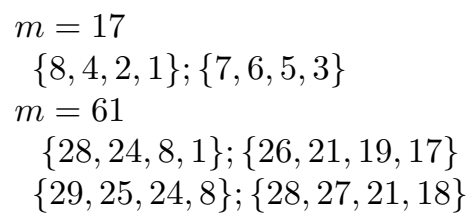

Like the case for 3-celestial configurations, we discovered some systematic families of 4-celestial configurations; these families have two parameters in addition to $q$.

- $2 q \#\{q-p, p, 2 p, q-4 r\} ;\{q-r, r, 2 r, q-4 p\}$

- $3 q \#\{q+p, q-p, p, 3 r\} ;\{q+r, q-r, r, 3 p\}$

- $6 q \#\{p, q+2 p, q+2 r, 2 q-p\} ;\{3 p, q-r, q-p, 2 q+r\}$

- $10 q \#\{p, 5 q-p, r, 5 q-r\} ;\{2 q, 4 q, 5 q-2 r, 5 q-2 p\}$

However, these are far from exhaustive.

\section{Other families}

\subsection{The general case where $m=2 q$}

We found a single family of systematic $2^{k+1}$-celestial configurations for even $m$ :

$$
2 q \#\left\{q-p, p, 2 p, 4 p, \ldots, 2^{k-1} p, q-2^{k} r\right\} ;\left\{q-r, r, 2 r, 4 r, \ldots, 2^{k-1} r, q-2^{k} p\right\}
$$

Note that the previously-known 3-celestial family with $m$ divisible by 2 , mentioned in Section 4 , is the case $k=1$ of this family, and the 4 -celestial case where $k=2$ is mentioned in Section 6.

We require the following lemma, whose proof is a straightforward induction on $k$; the base case is the trigonometric identity

$$
\sin (2 \theta)=2 \sin (\theta) \cos (\theta)
$$

in the case where $\theta=\frac{r \pi}{m}$.

Lemma 7.1. If $k, r, m$ are positive integers, then

$$
\sin \left(\frac{2^{k} r \pi}{m}\right)=2^{k} \sin \left(\frac{r \pi}{m}\right) \cos \left(\frac{2^{0} r \pi}{m}\right) \cos \left(\frac{2^{1} r \pi}{m}\right) \cdots \cos \left(\frac{2^{k-1} r \pi}{m}\right) .
$$


To see that the family in (7.1) works, it suffices to show that the cosine condition is satisfied. Note that

$$
\cos \left(\frac{(q-p) \pi}{m}\right)=\sin \left(\frac{p \pi}{2 q}\right)
$$

and

$$
\cos \left(\frac{\left(q-2^{k} r\right) \pi}{2 q}\right)=\sin \left(\frac{\left(2^{k} r\right) \pi}{m}\right) .
$$

Interpreting the left-hand cohort as a product of cosines and simpifying, we see that

$$
\begin{aligned}
& \text { LHS }=\cos \left(\frac{(q-p) \pi}{m}\right)\left[\cos \left(\frac{(p) \pi}{m}\right) \cos \left(\frac{(2 p) \pi}{m}\right) \cos \left(\frac{(4 p) \pi}{m}\right) \ldots\right. \\
&\left.\cos \left(\frac{\left(2^{k-1} p\right) \pi}{m}\right)\right] \cos \left(\frac{\left(q-2^{k} r\right) \pi}{m}\right) \\
&=\sin \left(\frac{(p) \pi}{m}\right)\left[\prod_{i=0}^{k-1} \cos \left(\frac{\left(2^{i} p\right) \pi}{m}\right)\right] \sin \left(\frac{\left(2^{k} r\right) \pi}{m}\right) \\
&=\frac{1}{2^{k-1}} \sin \left(\frac{2^{k} p \pi}{m}\right) \sin \left(\frac{\left(2^{k} r\right) \pi}{m}\right) \quad \text { (applying Lemma 7.1) }
\end{aligned}
$$

Since the left-hand and right-hand cohorts are symmetric in $p$ and $r$, the right-hand cohort also simplifies to

$$
\frac{1}{2^{k-1}} \sin \left(\frac{\left(2^{k} r\right) \pi}{m}\right) \sin \left(\frac{\left(2^{k} p\right) \pi}{m}\right)
$$

so the entire cohort satisfies (A3), the cosine condition.

\subsection{The general case where $m=10 q$}

Case 1: $4 \mid h$. There is an infinite family of $4 j$-celestial 4-configurations when $m=$ $10 q$ and $h=4 j$, of the form

$$
\begin{aligned}
& 10 q \#\left\{p_{1}, \ldots, p_{2 j}, 5 q-p_{1}, \ldots, 5 q-p_{2 j}\right\} ; \\
&\{\underbrace{2 q, \ldots, 2 q}_{j}, \underbrace{4 q, \ldots, 4 q}_{j}, 5 q-2 p_{1}, \ldots, 5 q-2 p_{2 j}\} .
\end{aligned}
$$

The 4-celestial family mentioned in Section 6 is the case $j=1$.

To see that this family satisfies the cosine condition, let $\phi=\frac{\pi}{10 q}$ and note that in the left-hand side, for each $i$, the pair

$$
\begin{aligned}
\cos \left(\left(5 q-p_{i}\right) \phi\right) \cos \left(p_{i} \phi\right) & =\frac{1}{2}\left(\cos \left(\frac{5 q \pi}{10 q}\right)+\cos \left(\left(5 q-2 p_{i}\right) \phi\right)\right) \\
& =\frac{1}{2} \cos \left(\left(5 q-2 p_{i}\right) \phi\right),
\end{aligned}
$$


so the entire left-hand side of the cosine condition becomes

$$
\left(\frac{1}{2}\right)^{2 j} \prod_{i=1}^{2 j} \cos \left(\left(5 q-2 p_{i}\right) \phi\right)=\frac{1}{4^{j}} \prod_{i=1}^{2 j} \cos \left(\left(5 q-2 p_{i}\right) \phi\right) .
$$

On the other hand, note that in the right-hand side, we have $\prod_{i=1}^{2 j} \cos \left(\left(5 q-2 p_{i}\right) \phi\right)$ already. Finally, note that each of the $j$ pairs

$$
\begin{aligned}
\cos \left(\frac{2 q \pi}{10 q}\right) \cos \left(\frac{4 q \pi}{10 q}\right) & =\frac{1}{2}\left[\cos \left(\frac{6 q \pi}{10 q}\right)+\cos \left(\frac{4 q \pi}{10 q}\right)\right] \\
& =\frac{1}{2}\left[\cos \left(\frac{3 \pi}{5}\right)+\cos \left(\frac{\pi}{5}\right)\right] \\
& =\frac{1}{2}\left(\frac{1}{4}(1-\sqrt{5})+\frac{1}{4}(1+\sqrt{5})\right) \\
& =\frac{1}{2}\left(\frac{1}{4}+\frac{1}{4}\right) \\
& =\frac{1}{4} .
\end{aligned}
$$

Thus, the entire right-hand side equals $\frac{1}{4^{j}} \prod_{i=1}^{2 j} \cos \left(\left(5 q-2 p_{i}\right) \phi\right)$, so the cosine condition is satisfied.

Case 2: $3 \mid h$. A similar infinite family exists when $m=10 q$ and $3 \mid h$ : if $h=3 j$, then

$$
\begin{aligned}
10 q \#\left\{p_{1}, 2 p_{1}, 5 q-2 p_{1}, \ldots, p_{j}, 2 p_{j}, 5 q-2 p_{j}\right\} & \\
& \left\{2 q, 4 q, 5 q-4 p_{1}, \ldots, 2 q, 4 q, 5 q-4 p_{j}\right\}
\end{aligned}
$$

is a $3 j$-celestial infinite family. Again, the 3-celestial family discussed in Section 4 is the case $j=1$.

The proof is similar to the previous family. Each right-hand triple $\left\{2 q, 4 q, 5 q-4 p_{i}\right\}$ becomes a multiplicand $\frac{1}{4} \cos \left(\left(5 q-4 p_{i}\right) \phi\right)$ in the cosine condition. On the other side, each left-hand triple $\{5 q-p, p, 2 p\}$ becomes

$$
\begin{aligned}
{[\cos ((5 q-p) \phi) \cos (p \phi)] \cos (2 p \phi) } & =\frac{1}{2}\left[\cos \left(\frac{5 q \pi}{10 q}\right)+\cos ((5 q-2 p) \phi)\right] \cos (2 p \phi) \\
& =\frac{1}{2} \cos ((5 q-2 p) \phi) \cos (2 p \phi) \\
& =\frac{1}{2}\left(\frac{1}{2}\left(\cos \left(\frac{5 q \pi}{10 q}\right)+\cos ((5 q-4 p) \phi)\right)\right) \\
& =\frac{1}{4} \cos ((5 q-4 p) \phi) .
\end{aligned}
$$

so the cosine condition is satisfied using any number of appropriate triples in the cohorts.

\section{Open questions for the classification of celestial 4-configurations}

Despite these classification results, there is clearly a lot about celestial configurations that is poorly understood. 
Question 8.1. Are the 3-celestial cohorts for $m=70, m=105$ sporadic, or are they members of an infinite family?

Question 8.2. Identify more systematic families of 3-celestial configurations for $m$ divisible by $30,42,60$. Are there systematic families for $m=90, m=120$ that are not multiples of smaller families? (Compare the situation for $m=6 q$ and $m=12 q$; there are families for $m=12 q$ that are different from the smaller $6 q$ families.)

Question 8.3. What's going on with the "sporadic family" of 3-celestial cohorts found for $m=66$ ? Does it generalize?

Question 8.4. In [16], M. Laczkovich discussed methods for identifying all rational solutions

$$
\sin \left(\pi p_{1}\right) \sin \left(\pi p_{2}\right) \sin \left(\pi p_{3}\right)=\sin \left(\pi q_{1}\right) \sin \left(\pi q_{2}\right) \sin \left(\pi q_{3}\right)
$$

If all solutions to this equation were known, then, using techniques similar to the classification of 2-celestial configurations, it should be possible to classify all 3-celestial configurations. However, Laczkovich's results are not presented in an obviously accessible way for this purpose. How can his results be used to classify 3-celestial configurations, or at least to identify new families?

For $m \leq 120$, there are no 3-celestial configurations for prime $m$. However, there is one 4-celestial cohort with $m=17$, and there are two 4-celestial cohorts for $m=61$.

Question 8.5. Are there any 3-celestial configurations for prime $m$ or prime powers $m$ ? Are there other 4-celestial configurations for prime $m$ ? prime powers?

Question 8.6. Identify new systematic families of 4-celestial configurations, especially for $m$ divisible by 6 .

Question 8.7. Given a known family, is there a general technique to apply "cosine trickery" to produce a different family that has related parameters? That is, from a single infinite family, are there a number of "combinatorially related" infinite families that can be produced?

Question 8.8. For $h=3$ and $h=4$, systematic families for $m=2 q, 3 q$ and $10 q$ have been identified. Two of these $(m=2 q$ and $m=10 q)$ correspond to infinite families for infinitely many $h$. Is there a corresponding infinite family of $h$-celestial configurations for $m=3 q$ ? Are there infinite families of $h$-celestial configurations for $m=2 q$ when $h$ is not a power of 2? Are there more infinite families when $m=10 q$ ?

\section{Data}

Raw data is available at

https://sites.google.com/a/alaska.edu/lwberman/.

It was produced using Mathematica.

\section{Acknowledgements}

The authors thank the Ursinus College Summer Fellows Program for funding the initial part of this project. 


\section{References}

[1] L. W. Berman, A characterization of astral $\left(n_{4}\right)$ configurations, Discrete Comput. Geom. 26 (2001), 603-612.

[2] L. W. Berman, Even astral configurations, Electron. J. Combin. 11 (2004), Research Paper 37, 23 pp. (electronic).

[3] L. W. Berman, Movable $\left(n_{4}\right)$ configurations, Electron. J. Combin. 13 (2006), Research Paper 104, 30 pp. (electronic).

[4] L. W. Berman, Some results on odd astral configurations, Electron. J. Combin. 13 (2006), Research Paper 27, 31 pp. (electronic).

[5] L. W. Berman and N. A. Burtt, A new construction for symmetric $(4,6)$-configurations, Ars Math. Contemp. 3 (2010), 165-175.

[6] L. W. Berman and B. Grünbaum, Deletion constructions of symmetric 4-configurations. Part I, Contrib. Discrete Math. 5 (2010), 18-33.

[7] A. Betten, G. Brinkmann and T. Pisanski, Counting symmetric configurations $v_{3}$, in: Proceedings of the 5th Twente Workshop on Graphs and Combinatorial Optimization (Enschede, 1997), volume 99, 2000 pp. 331-338, doi:10.1016/S0166-218X(99)00143-2.

[8] M. Boben and T. Pisanski, Polycyclic configurations, European J. Combin. 24 (2003), 431457, doi:10.1016/S0195-6698(03)00031-3.

[9] B. Grünbaum, Configurations, 1991, unpublished course notes.

[10] B. Grünbaum, Astral ( $\left.n_{k}\right)$ configurations, Geombinatorics 3 (1993), 32-37.

[11] B. Grünbaum, Astral $\left(n_{4}\right)$ configurations, Geombinatorics 9 (2000), 127-134.

[12] B. Grünbaum, Configurations of points and lines, in: The Coxeter legacy, Amer. Math. Soc., Providence, RI, pp. 179-225, 2006.

[13] B. Grünbaum, Musings on an example of Danzer's, European J. Combin. 29 (2008), 1910 1918, doi:10.1016/j.ejc.2008.01.004.

[14] B. Grünbaum, Configurations of points and lines, volume 103 of Graduate Studies in Mathematics, American Mathematical Society, Providence, RI, 2009.

[15] B. Grünbaum and J. F. Rigby, The real configuration (214), J. London Math. Soc. (2) 41 (1990), 336-346, doi:10.1112/jlms/s2-41.2.336.

[16] M. Laczkovich, Configurations with rational angles and trigonometric Diophantine equations, in: Discrete and computational geometry, Springer, Berlin, volume 25 of Algorithms Combin., pp. 571-595, 2003.

[17] D. Marušič and T. Pisanski, Weakly flag-transitive configurations and half-arc-transitive graphs, European J. Combin. 20 (1999), 559-570, doi:10.1006/eujc.1999.0302.

[18] G. Myerson, Rational products of sines of rational angles, Aequationes Math. 45 (1993), 70-82, doi:10.1007/BF01844426. 